\title{
A NOTE ON THE CONSTRUCTION OF SIMPLY-CONNECTED 3-MANIFOLDS AS BRANCHED COVERING SPACES OF $S^{3}$
}

\begin{abstract}
JOAN S. BIRMAN
Abstract. Let $K$ be a knot in $S^{3}$, and let $\omega: \pi_{1}\left(S^{3}-K\right) \rightarrow \Sigma_{n}$ be a transitive representation into the symmetric group $\Sigma_{n}$ on $n$ letters. The pair $(K, \omega)$ defines a unique closed, connected orientable 3-manifold $M(K, \omega)$, which is represented as an $n$-sheeted covering space of $S^{3}$, branched over $K$. A procedure is given for representing $M(K, \omega)$ by a Heegard splitting, and a formula is given for computing the genus of that Heegard splitting of $M(K, \omega)$. This formula is then applied to the 3-sheeted irregular covering spaces studied by Hilden (Bull. Amer. Math. Soc. 80 (1974), 1243-1244) and Montesinos (Bull. Amer. Math. Soc. 80 (1974), 845-846), and, also, Tesis (Univ. de Madrid, 1971) to show that these particular covering spaces cannot yield counterexamples to the Poincare Conjecture if the branch set has bridge number $<4$.
\end{abstract}

In [3] R. H. Fox described a method for constructing 3-manifolds, and in particular for seeking possible counterexamples to the 3-dimensional Poincare Conjecture. Fox considers a knot $K$ in $S^{3}$, together with a transitive representation $\omega$ of $G=\pi_{1}\left(S^{3}-K\right)$ into the symmetric group $\Sigma_{n}$ on $n$ letters. The pair $(K, \omega)$ defines a unique closed, connected orientable 3-manifold $M=M(K, \omega)$, which is constructed by taking $n$ copies of $S^{3}$, labeled 1 through $n$, and gluing them together along a surface spanned by $K$ according to $w$. A presentation may be found for $\pi_{1} M$ from the data given by $w$, and if $\pi_{1} M=1$ and $K$ is sufficiently complicated, one is lead to question whether $M$ might not be a homotopy sphere distinct from $S^{3}$. Quoting Fox, "one can find bushel baskets of ... simply connected ...3-manifolds this way. No doubt most of them, and possibly all of then, are actually $S^{3}$, but ...".

Fox's construction has generated new interest recently, because of results of Hilden [4], [5], and Montesinos [7], [8], who established that every closed, orientable 3-manifold may be represented as $M(K, \omega)$ for some knot $K$ and some transitive representation $w$ of the group $G$ into the symmetric group $\Sigma_{3}$ on 3 letters. A second result of Montesinos [6] makes these particular covering spaces even more appealing, for Montesinos has discovered modifications which are possible in the branch set without altering the topology of $M(K, \omega)$. These modifications cause the branch set to change dramatically before one's eyes, altering not only knot type, but also changing knots to links, and splitting a link into two or more unlinked components, thus suggesting the possibility of systematic simplification to obtain a "best" choice for the branch set. The

Received by the editors October 7, 1974 and, in revised form, December 6, 1974.

AMS (MOS) subject classifications (1970). Primary 55A10, 55A40, 55A25.

Key words and phrases. 3-manifold, homotopy sphere, branched covering space, knot, Heegard splitting.

${ }^{1}$ Supported in part by NSF Grant No. GP 32843. 
hope would be that if a simply connected 3-manifold could be associated with a branch set that admitted no further Montesinos simplifications, then that manifold would stand a good chance of being distinct from $S^{3}$, since $S^{3}$ may be represented as a 3 -fold irregular covering of $S^{3}$ branched over the trivial link of 2 components. The purpose of this note is to point out that there is, alas, an inherent difficulty in this approach, which makes it unlikely that one will locate a counterexample by the method described above.

Note first that the procedure depends first on the computation of $\pi_{1}(M)$, which will be defined by a presentation; see [3]. The number of generators and relations in that presentation will increase as the knot increases in complexity, so that for excessively complicated knots it will, in general, be difficult (or impossible) to decide whether $\pi_{1}(M)=1$. A crucial question to ask, then, is which are the simplest knots which stand any chance of providing the example we seek?

We consider a special representation of the branch set as a subset of the 3sphere $S^{3}$. Let $D$ be a 3-ball, and let $A=A_{1} \cup \cdots \cup A_{b}$ be a set of $b$ unknotted, unlinked arcs properly imbedded in $D$, with $\partial A \subset \partial D$. Let $\left(D^{\prime}, A^{\prime}\right)$ be a copy of $(D, A)$. If we now identify $(D, A)$ with $\left(D^{\prime}, A^{\prime}\right)$ by a sewing map $\phi(\partial D, \partial A) \rightarrow\left(\partial D^{\prime}, \partial A^{\prime}\right)$ then the identification space $K=A \cup_{\phi} A^{\prime}$ is readily seen to be a knot or link in $S^{3}=D \cup_{\phi} D^{\prime}$. It is known (see, for example, [2]) that every knot or link admits such a representation, for an appropriate choice of $\phi$, and also that the bridge number of the knot is precisely the minimum value of the integer $b$. Consider $n$ indexed copies of $S^{3}$, each represented as above in the form $D \cup_{\phi} D^{\prime}$, and each containing the distinguished subset $K=A \cup_{\phi} A^{\prime}$. The representation $w$ then tells us how to glue together these copies, with distinct sheets of the covering space coming together along a spanning surface for $K$, in order to construct $M$.

The subset of $M$ which is formed by identifying the $n$ copies of $D$ (respectively $D^{\prime}$ ) is a handlebody and its surface $F$ (respectively $F^{\prime}$ ) is a closed orientable surface of some genus $g$, i.e., a Heegaard surface in $M$. The surface $F$ is represented as an $n$-fold branched covering space of $S^{2}$, branched over the $2 b$ points of $\partial A \subset S^{2}$.

Let $B$ denote the sum of the orders of all the branch points on $F$. Then, by a well-known formula (see, for example, [9, p. 275]) the genus $g_{0}$ of $F$ is given by

$$
g_{0}=B / 2-n+1
$$

In a $n$-sheeted covering space the sum of the orders of the branch points of $F$ which cover a given branch point $z_{0} \in S^{2}$ is at most $n-1$, and at least 1 . Since our covering spaces have $2 b$ branch points, it then follows that

$$
b-n+1 \leqq g_{0} \leqq(b-1)(n-1) \text {. }
$$

The cyclic covering spaces correspond to the upper bound, and the HildenMontesinos covering spaces to the lower bound. ${ }^{2}$ Since $g \leqq g_{0}$, one may thus see that in the 3-fold irregular coverings of Hilden and Montesinos,

$$
g \leqq b-2 \text {. }
$$

Formula (3) now indicates that if $K$ has bridge number 2 then $M$ would have Heegaard genus 0 , hence $M$ can only be $S^{3}$. If $K$ is a 3-bridge knot, then $M$

\footnotetext{
2 This is so because exactly 2 sheets come together at each branch point.
} 
will have Heegaard genus 1 , and in this case (by classical results) $H_{1}(M)$ $=1$ implies $M \approx S^{3}$. (Note that we have already exhausted all the knots in the Reidemeister table, since all the latter have bridge number $\leqq 3$.) Suppose next that $K$ has bridge number 4 . Then $M$ has Heegaard genus 2 , and by results of the author and Hilden [1], [2], $M$ may also be represented as $M\left(K^{\prime}, t\right)$ for some 3-bridge knot $K^{\prime}$ and the associated unique representation $t$ of $\pi_{1}\left(S^{3}-K^{\prime}\right)$ onto $\Sigma_{2}$. It then follows from results of Waldhausen [10] that $M \approx S^{3}$ if and only if $K^{\prime}$ is the trivial knot type. Thus, if one hopes to find a counterexample to the Poincaré Conjecture by examining 3-fold coverings of $S^{3}$ branched over 4-bridge knots, with the goal of applying the Montesinos moves in an attempt to simplify the branch set, then (at best) if one is clever in the application of these moves, one will ultimately reduce the knot to a 3 bridge knot represented on $\Sigma_{2}$, which may or may not be trivial. But, since the calculation of $\pi_{1} M$ is simpler for 2-fold coverings of $S^{3}$ branched over 3bridge knots than for 3-fold coverings branched over 4-bridge knots, and also since one knows that if one starts with a nontrivial 3-bridge knot then its 2fold cover cannot be $S^{3}$, one might as well have started the search with 2 -fold coverings and 3-bridge knots in the first place! Moreover, the 2-fold (and more generally the cyclic) covering spaces of $S^{3}$ branched over knots do not seem to be very likely candidates for our sought-for counterexample because of the many special symmetries which they exhibit (see, for example, $[2, \S 6])$. Thus, life does not begin to get really interesting for these 3-fold covering spaces until the branch set has bridge index 5 , which places the knots outside all the standard tables and makes the entire procedure a very unappealing and timeconsuming calculation, if, indeed, it is possible to decide whether $\pi_{1} M=1$ for such an $M$. Similar phenomena have characterized many of the attempts I have seen to seek counterexamples to the Poincare Conjecture, however this does not in any way indicate that they do not exist.

\section{REFERENCES}

1. J. S. Birman and H. M. Hilden, The homeomorphism problem for $S^{3}$, Bull. Amer. Math. Soc. 79 (1973), 1006-1010. MR 47 \#7726.

2. - Heegaard splittings of branched coverings of $S^{3}$, Trans. Amer. Math. Soc. 213 (1975), 315-352.

3. R. H. Fox, Construction of simply connected 3-manifolds, Topology of 3-Manifolds and Related Topics (Proc. Univ. of Georgia Inst., 1961), Prentice-Hall, Englewood Cliffs, N. J., 1962, pp. 213-216. MR 25 \#3539.

4. H. M. Hilden, Every closed, orientable 3-manifold is a 3-fold branched covering space of $S^{3}$, Bull. Amer. Math. Soc. 80 (1974), 1243-1244.

5. - Three-fold branched coverings of $S^{3}$, Amer. J. Math. (to appear).

6. J. M. Montesinos, Sobre la conjetura de Poincaré y los recubridores ramificados sobre un nudo, Tesis, Universidad de Madrid, 1971.

7. - A representation of closed orientable 3-manifolds as 3-fold branched coverings of $S^{3}$, Bull. Amer. Math. Soc. 80 (1974), 845-846.

8. - Three-manifolds as 3-fold branched covers of $S^{3}$, Bull. Amer. Math. Soc. 80 (1974), 845-846.

9. G. Springer, Introduction to Riemann surfaces, Addison-Wesley, Reading, Mass., 1957. MR 19, 1169.

10. F. Waldhausen, Über Involution der 3-Sphäre, Topology 8 (1969), 81-91. MR 38 \# 5209.

Department of Mathematics, Barnard College of Columbia University, New York, NEW YORK 10027 